\title{
Gravitational Time Dilation Derived from Special Relativity and Newtonian Gravitational Potential
}

\author{
Rickey W. Austin, \\ (PhD Candidate, NC A\&T, Greensboro, NC, USA) \\ Physics Based Modeling Group, \\ Applied Research Associates (ARA), Suite, Raleigh, NC USA
}

doi: 10.19044/esj.2016.v13n3p447 URL:http://dx.doi.org/10.19044/esj.2016.v13n3p447

\begin{abstract}
Einstein was known for often presenting "gedanken" or thought experiments (Miller, 1999). This paper is such an exercise. Time dilation in Special Relativity is based on the derived value of $\gamma$ (a scalar value) (Einstein, 1905). $\quad \gamma$ will be calculated as a function of relativistic kinetic energy, allowing time dilation to become a function of relativistic kinetic energy. With the new methodology constructed, a "gedanken" experiment is considered. Can a time dilation function be derived, using Newtonian gravitational potential energy in the same manor kinetic energy was used in Special Relativity? This paper carries out the derivation and compares the results with General Relativity's Schwarzschild solution. The what if thought experiment provides a first order accuracy to GR's Schwarzschild solution.
\end{abstract}

Keywords: Gravitational Time Dilation, Special Relativity

\section{Introduction:}

In Special Relativity the time component in standard configuration (Muirhead, 1973) is translated via

$t^{\prime}=\gamma\left(t-\frac{v x}{c^{2}}\right)$

from the Lorentz transformation (Poincare, 1905) where $\gamma$ is the Lorentz boost, $\mathrm{c}$ is the speed of light in a vacuum, $\mathrm{v}$ is the relative velocity between frames, $t$ is the time in the rest frame and $t$ is the time in the frame in motion. With no change in the $\mathrm{x}$ direction, equation (1) reduces to $t^{\prime}=\gamma t$

Equation (2) transforms a time interval as observed from a frame at rest to the time interval as observed in a frame in uniform motion. 
Another approach to deriving $\gamma^{\prime}$ s value is to consider relativistic kinetic energy (Einstein,1905) (Resnick,Halliday, 1985).

$T=E_{0}(\gamma-1)$

Where $\mathrm{T}$ is the kinetic energy of the moving particle with rest energy $E_{0}=m c^{2}$, where $\mathrm{c}$ is the speed of light in a vacuum. Divide both sides by rest energy and rearrange:

$\gamma=1+\frac{T}{E_{0}}$

Substituting equation (4) into equation (2)

$t^{\prime}=\left(1+\frac{T}{E_{0}}\right) t$

Equation (5) is a derived transformation for time between two observers based on the difference in kinetic energy between two observers. The difference is between a stationary particle in a rest frame, where kinetic energy is zero, and a particle at rest in a moving frame where kinetic energy is greater than zero (as observed from the rest frame). With an energy based time dilation, Newtonian gravitational potential energy is considered.

\section{History of Newtonian Gravitational Time Dilation:}

Newtonian classical physics does not predict time dilation between observers (Newton, 1689). Both time and space are assumed absolute to all observers. In 1887 Michelson-Morley's (Michelson, 1887) conducted an experiment which results lead to Poincare' and Lorentz mathematically considering time as not absolute (Poincare', 1905)( Lorentz, 1892). Shortly thereafter in 1905 Einstein presented his theory of Special Relativity. In his theory, time, previously considered absolute, was predicted to be relative between observers depending on their relative velocity and the consistency of the speed of light between all observers (Einstein, 1905).

Einstein, roughly ten years later, presented his theory of General Relativity (GR) and introduced the concept of mass-energy causing a curvature in space-time (Einstein, 1914). By combining Riemannian geometry and a stress energy tensor, Einstein changed the accepted model of gravity from a classical central force to a model based on the curvature of space-time (Wheeler, 1973, pages 289-303). From the model of GR, field equations are derived. The solution to the field equations is provided by a metric (Carroll, 2007, pages 628-633). The first published solution to Einstein's field equations was the Schwarzschild metric (Hartle, 2003, page 186). The Schwarzschild metric is an exact solution to Einstein's equation for a non-rotating, non-charged mass (Schutz, 1990, page 275). The Schwarzschild solution accurately predicted the perihelion advance of mercury, light curvature and time dilation due to energy (mass and momentum) (Stephani, 1982. Pages 99-120). 
With the advent of Special and General Relativity, physics was given two new categories; classical (sometimes referred to as Newtonian) and relativistic (Thorton, 2002, pages 27-73). Relativistic most often is considered in velocity induced time dilation, but gravitational time dilation is also referred to as a relativistic effect. The distinction comes from absolute time in classical mechanics and none absolute time in relativistic mechanics.

Via researching the university's library, online article references and Google Scholar, no previous published work comparing SR kinetic energy and Newtonian potential energy for deriving gravitational time dilation could be found. Online lecture notes from Gary Oas, PhD at Stanford University discuss a classical energy approach to using Newtonian gravity and SR. His approach does not consider the similarity of SR's derivation as a basis for constructing a Newtonian gravitational potential. None-the-less it does have a mixture of Newtonian gravity and SR to derive a matching Schwarzschild time dilation in a weak gravitational field (Oas, 2012) and should be mentioned here.

\section{Newtonian Gravitational Potential:}

Classical Newtonian gravitational potential energy (NGPE) is commonly derived by integrating Newtonian gravitational force over a distance (Carroll, 2008). Newtonian gravitational force is

$$
F_{G}=\frac{G M_{1} M_{2}}{r^{2}}
$$

Where $\mathrm{G}$ is Newton's gravitational constant, $M_{1}$ and $M_{2}$ are point masses whose centers are separated by radial distance r. NGPE is the potential energy when changing position relative to the center of mass (a change in r). To calculate the change in potential energy, integrate the force over the change in $\mathrm{r}$ :

$$
V_{G}=\int_{a}^{b} \frac{G M_{1} M_{2}}{r^{2}} d r
$$

Where $\mathrm{a}$ and $\mathrm{b}$ are radial distances between the center of the two masses. As the two masses move toward or away from each other, the gravitational potential energy between them changes.

\section{Gravitational Time Dilation Hypothesis:}

After briefly reviewing NGPE a hypothesis is set forth. Gravitational time dilation can be derived from NGPE in the same manor $\gamma$ was derived from kinetic energy. Before applying the derivation from kinetic energy to NGPE, first consider a special case of NGPE.

\section{Special Case of NGPE:}

A special case of NGPE exist when the potential energy is calculated using the following setup. 
- Move a point mass from its location to infinity

- The mass is moved to infinity through the potential it formed at its original point in space

In plain English, the point mass is fixed in space and an equal mass is moved from its radius to infinity. The mathematical calculation is equation (7) where $M_{1}=M_{2}$ and $M_{1}$ is fixed in space. Thus this special case of NGPE is

$$
V_{G}=\int_{\text {radius }}^{\infty} \frac{G M^{2}}{r^{2}} d r \stackrel{\text { yields }}{\longrightarrow}-\frac{G M^{2}}{r}
$$

Equation (8) is a calculation of the NGPE required to move an equal mass from its location to infinity under its own Newtonian gravitational potential. It is a unique magnitude for any mass with a fixed radius.

\section{Apply SR's derivation of $\boldsymbol{\gamma}$ to NGPE:}

Consider the hypothesis from section Gravitational Time Dilation Hypothesis using $V_{G}$ to obtain a scalar time dilation in the same way $\mathrm{T}$ was used in calculating $\gamma$. Classically the total energy of a mechanical system is the sum of kinetic energy (T) and potential energy (V) (Fishbane, 2005). Assume the total energy of a system correlates to a time dilation. Classically the total energy is

$$
E_{\text {total }}=T+V
$$

Using equation (4) and substituting the total energy from equation (9) for $\mathrm{T}$, the equation becomes:

$$
\text { Time Dilation Scalar }=1+\frac{T+V}{E_{0}}
$$

If only considering NGPE for $\mathrm{V}$ and no kinetic energy $(\mathrm{T}=0)$, equation (10) becomes:

$$
\varphi=1+\frac{V_{G}}{E_{0}}
$$

Where $\varphi$ is the gravitational time dilation scalar. Substituting equation (8) into equation (11)

$$
\varphi=1-\frac{G M^{2}}{r M c^{2}} \stackrel{\text { yields }}{\longrightarrow} 1-\frac{G M}{r c^{2}}
$$

Following the time dilation derivation based on kinetic energy, the hypothesized gravitational time dilation would follow equation (2)'s method, except using NGPE's derived value of $\varphi$ instead of $\gamma$.

$$
t^{\prime}=\varphi t
$$

Where $t$ is the time interval at infinity and $t^{\prime}$ is the time interval at $\varphi$ 's calculated radial distance from the point mass.

The time dilation is calculated at a static point in space about a static point mass. There are no other causes of time dilation considered (relative motion or test mass). With the new hypothesis derived in equation (13), how 
does it compare to General Relativity’s Schwarzschild solution? Before comparing, inertial and non-inertial frames need to be discussed.

\section{Inertial Frames in a Gravitational Potential:}

A major difference between Special Relativity’s transformation and a gravitational potential transformation is inertial frames. A frame in a gravitational potential is no longer an inertial frame, but an accelerated frame (Tocaci, 1984). For this thought experiment walk-through, the following assumptions are made:

- Proper time is located at an infinite distance from the mass where the potential no longer has a measurable effect

- Proper time is located in an inertial frame

- The accelerated frame's measurements are at an instantaneous velocity

With these assumptions, the transformation takes place in an accelerated frame at a given radius from the point mass generating the gravitational potential field to an inertial frame infinitely far away. Once the instantaneous velocity of the accelerated frame is obtained, an inertial frame can be constructed with the same constant velocity as the accelerated instantaneous velocity. By considering the inertial frame with a constant velocity equal to the instantaneous velocity of the accelerated frame, and using this frame for all transformations, criteria for inertial frame transformations are met. There are obvious differences when considering mechanics, forces, and the like between an instantaneous accelerated frame and an inertial frame. This thought experiment is only interested in the time dilation transformation. As such, the inertial frame with the same constant velocity as the instantaneous velocity would share the same time dilation at a given instance as the accelerated frame.

\section{$\varphi$ Compared to General Relativity's Schwarzschild's Time Dilation:}

When considering a fixed mass in space, with no spin or charge within General Relativity, the Schwarzschild metric (previously discussed) is a well-known solution to Einstein's field equations (Antoci, 2003). The Schwarzschild metric predicts time dilation between two points in space as (When caused only by the curvature of space-time due to a static neutral mass. It should be noted there is no radial angle change between the two locations in space. Thus only the radial distance is changing, allowing for the following temporal transformation).

$$
t^{\prime}=\left(\sqrt{1-\frac{2 G M}{r c^{2}}}\right) t
$$


Where $\mathrm{t}$ is located within the gravitational field and $\mathrm{t}^{\prime}$ is the Schwarzschild coordinate time located an infinite distance from the gravitational potential (Carroll, 2008 pages 630-640).

Substitute

$$
x=\frac{M}{r}
$$

into equation (14) and calculate a Taylor series on $\mathrm{x}$. The expansion point is about 0 to the second order.

$$
\sqrt{1-\frac{2 G x}{c^{2}}} \stackrel{\text { yields }}{\longrightarrow} 1-\frac{G x}{c^{2}}-\frac{G^{2} x^{2}}{2 c^{4}}+O\left(x^{3}\right)
$$

Substitute equation (15) into equation (12), the NGPE derived transformation,

$$
\varphi=1-\frac{G x}{c^{2}}
$$

Equations (16) and (17) are equal to first order. The newly derived transformation value of $\varphi$, (equation 12) is an energy based time dilation due to a gravitational potential energy. The transformation is first order accurate to current accepted theory. The thought experiment allows the transformation of time, derived from SR's kinetic energy (equation 4) to be applied to NGPE. The outcome is minimum first order accuracy to current theory.

It should be noted this is a transformation comparison between NGPE and the Schwarzschild GR solution. The Schwarzschild solution is a geometrical solution and a special case (radial coordinate change only) is used to form a transformation matching the derived NGPE transformation conditions. In no way should one consider the NGPE transformation a substitute or equivalence to GR or the Schwarzschild solution. It is the standard accepted transformation by which the NGPE is compared to.

\section{Conclusion:}

Deriving an equation to calculate time dilation from kinetic energy provided a hypothesized methodology to calculate gravitational potential energy's time dilation. Deriving the equation using a special case of Newtonian Gravitational Potential Energy equation (8), a method to calculate time dilation due to NGPE was constructed. When the newly derived method is compared to General Relativity's Schwarzschild's solution (for a radial only change in position), the time dilation is found to be equal to first order via Taylor expansion. This is not an attempt to replace GR, it is a simple exercise in a hypothesis of how time dilation, as a function of energy (kinetic) would compare to a similarly constructed method with NGPE. The results show a first order accuracy to accepted theory. 
The author would like to say thank you to the reviewers who, through their experience offered clarity, depth and professionalism to the final version of the paper.

\section{References:}

1. Miller, Author I. (1999) Einstein's First steps toward general relativity gedanken experiments and axiomatics. Physics in Perspective.

2. Einstein, Albert (1905) On the electrodynamics of moving bodies. Annalen der Physik.

3. Muirhead H. (1973) The Special Theory Of Relativity. Halsted Press.

4. Poincare' Henri (1905). On the dynamics of the electron. Sitzungsberichte der Deutschen Akademie der Wissenschaften zu BerlinComptes rendus hebdomadaires des seances de l'Academie des sciences.

5. Halliday, David., \& Resnick, Robert.(1985) Basic Concepts In Relativity And Early Quantum Theory. John Wiley and Sons, Inc., 2 edition.

6. Carroll., Bradley., Ostlie., Dale. (2007) An Introduction To Modern Astrophysics. Pearson\, second edition\, 2007.

7. Tocaci, Emil \& Kilmister, Clive William. (1984) Relativistic Mechanics, Time and Inertia. Springer, first edition.

8. Antoci, S. \& Liebscher (2003). On the gravitational Field of a mass point according to einstein's theory by $k$. schwarzschild. General Relativity and Gravitation, 35:945.

9. Hartle, James B., (2003), Gravity An introduction to Einstein's General Relativity, Addison Wesley

10. Schutz, Bernard F., (1995) A first course in general relativity, Cambridge University Press

11. Stephani, Hans (1982), General Relativity An introduction to the theory of the gravitational field, Cambridge University Press

12. Thornton, Stephen T. \& Rex, Andrew (2002), Modern Physics for scientists and engineers, Brooks/Cole

13. Einstein, Albert (1914), Bases physiques d'une théorie de la gravitation, Archives des sciences physiques et naturelles (ser. 4), 37, 5-12

14. Wheeler, John Archibald., Misner, Charles W., Thorne, Kip S., (1973), Gravitation, W.H. Freeman and Company

15. Newton, Isaac, (1689), Philosophiae Naturalis Principia Mathematica

16. Michelson, Morley (1887) On the relative motion of the earth and the luminiferous ether. American Journal of Science, 34 
17. Lorentz, Hendrik Antoon (1892), The relative motion of the earth and the aether. Zittingsverlag Akad, 1:74

18. Fishbane, Gasiorowicz, Thornton (2005), Physics for Scientists and Engineers with Modern Physics, Prentice Hall

19. Oas, Gary (2012), Lectures on General Relativity, Standford University,

http://web.stanford.edu/ oas/SI/SRGR/notes/SRGRLect9B_2013.pdf 